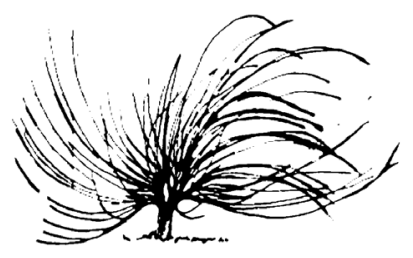

\title{
El quehacer del científico: Una perspectiva crítica desde referentes psicológicos
}

\author{
Manuel Villarruel-Fuentes ${ }^{1}$ \\ Tecnológico Nacional de México-Instituto Tecnológico de Úrsulo Galván \\ México \\ m.villarruel@itursulogalvan.edu.mx
}

\begin{abstract}
Resumen
La ciencia como pensamiento organizado se encuentra sujeta a normas y principios que se plasman en la práctica cotidiana del sujeto que piensa y hace investigación. Alineado con ello, es posible identificar comportamientos irracionales denominados sesgos cognitivos, los cuales afectan el resultado de todo proceso de indagación y generación de conocimiento. Con base en ello, se presenta un análisis crítico sobre aquellos sesgos que afectan el pensamiento del personal científico que hace y enseña ciencia, en busca de planteamientos conceptuales que fundamenten nuevos problemas de investigación.
\end{abstract}

Palabras clave: Sesgos cognitivos, razonamientos, ciencia, investigación, escolar.

\begin{abstract}
Science as organized thought is subject to rules and principles that are embodied in the daily practice of the subject who thinks and does research. Aligned with this, it is
\end{abstract}

Recibido: 9 de julio de 2018. Aprobado: 10 de abril de 2019 .

http://dx.doi.org/10.15359/rep.14-1.3

1 Profesor-Investigador. Líder del Cuerpo Académico “Cultura Académica y Desarrollo Social Sustentable". Línea de Investigación: "Educación, ciencia, sociedad y tecnología para un desarrollo humano sostenible". Miembro del Sistema Nacional de Investigadores en México y del Consejo Mexicano de Investigación Educativa". 
possible to identify irrational behaviors called cognitive biases, which affect the outcome of any process of inquiry and generation of knowledge. Based on this, a critical analysis is presented on those biases that affect the thinking of the scientist who makes and teaches science, in search of conceptual approaches that support new research problems.

Keywords: Cognitive biases, reasoning, science, research, school.

\section{Planteamiento del problema y fundamentación teórica}

A partir de las viejas tradiciones intelectuales predominantes en el pensamiento científico occidental, consolidado por un Modernismo distinguido por el razonamiento formal, de carácter deductivo-inductivo, sustentado en la evidencia empírico-analítica, donde el ascetismo metodológico descansa en la experimentación y la lógica del error, se explica el paradigma educativo dominante en la educación escolar del siglo XX y lo que va del XXI, particularmente en la denominada "ciencia escolar".

Más allá de las formas de concebir las premisas de una ciencia que atiende los aspectos propios de cada campo disciplinar, con el propósito de generar y validar el núcleo de conocimiento que le respalda, de lo que se trata es de pensar la ciencia como una actividad social, que hunde sus raíces en la cultura de los pueblos, en sus imaginarios colectivos, en las representaciones sociales que definen las imágenes, creencias y simbolismos que les son propios, los que al paso del tiempo han caracterizado su devenir histórico. Ferreira (2017a), desde una perspectiva antropológica, despeja este aspecto al indicar:

Partimos de una concepción que entiende la ciencia como una actividad, una actividad social vertebrada por prácticas reflexivas situadas contextualmente. Ello conjugado con la aceptación de que nuestras disposiciones y predisposiciones para la acción e interacción se fundamentan en la incorporación de un habitus, según el cual sentido práctico y sentido representacional están firmemente imbricados, nos lleva a asumir que, en esa práctica social que es "hacer ciencia", lo que hace el científico (actividad 
práctica) no es distinto del producto resultante de su actuación (representación objetiva del mundo real). Lo cual podemos reformular afirmando que la presunta universalidad de los productos del conocimiento científico no sería sino una construcción más de su práctica cotidiana (expresión de esa imbricación entre sentido práctico y sentido representacional). (p. 42)

Se trata, ni más ni menos, que de una ciencia que dota a comunidades muy específicas de los denominados "campos de reproducción simbólica", no exentos del juego del poder donde sus integrantes buscan ganar los espacios de opinión pública a partir del dominio de las percepciones sociales. Bourdieu (2000), desde la sociología, lo expresa con claridad cuando señala:

El campo científico, como sistema de relaciones objetivas entre posiciones adquiridas (en las luchas anteriores), es el lugar (es decir, el espacio de juego) de una lucha competitiva que tiene por desafío específico el monopolio de la autoridad científica, inseparablemente definida como capacidad técnica y como poder social, o, si se prefiere, el monopolio de la competencia científica que es socialmente reconocida a un agente determinado, entendida en el sentido de capacidad de hablar e intervenir legítimamente (es decir, de manera autorizada y con autoridad) en materia de ciencia. (p. 12)

Sobre esta vertiente es necesario reconocer que dicha ciencia toma dimensiones comunes, direccionadas desde la individualidad de los sujetos, quienes dotan de sentido las prácticas dominantes y sucedáneas de las comunidades científicas. Desde este referente, es posible entender la influencia que tiene la psique de quienes realizan investigación con los cánones y axiomas que la ciencia reglada impone. A partir de estas premisas, pensar la ciencia desde los espacios escolares exige atender la figura del personal docente-experto, entendido como aquel que encontrándose alfabetizado científicamente, enseña ciencia a estudiantes-aprendices, en un proceso didáctico que replica las formas de concebir lo real, en un marco de aprendizajes guiados, sustentados por la participación en proyectos que actúan como escenarios donde se recrea el quehacer científico y se reafirman los principios legaliformes 
de la ciencia normal (Villarruel-Fuentes, 2013). Los grupos expertos, a decir de Ferreira (2007b), son los que promueven, sancionan, validan $\mathrm{y}$, en su momento, crean, reproducen o sustituyen los paradigmas científicos que componen el sustrato que faculta una visión particular del mundo y del proceder que despliegan los mismos grupos expertos en el intento de conocerlos y recrearlos.

Bartolucci (2017) destaca la importancia de los "productos" desde una visión sociológica, cuando puntualiza:

Si bien la atención sociológica se dirige hacia los "productos", lo hace atendiendo a la serie de acciones que participan en el proceso de constitución colectiva de su realidad, concibiéndola como el entrecruzamiento de acciones materiales y simbólicas que resultan sustantivas para quienes comparten un marco de referencia tan peculiar como el científico. Lo técnico no está separado ni es ajeno a lo social, y mucho menos cabe suponer que lo primero sea lo esencial de la ciencia y lo segundo algo contingente. (p. 28)

Como se observa, no se trata de cualquier tipo de productos, sino de unos en especial. Son productos científicos que reúnen "coherencia lógica, pertinencia teórica, relevancia temática, consistencia empírica, originalidad disciplinaria y congruencia argumental, no sólo como reglas cognoscitivas sino como formas simbólicas de materializar los principios éticos consustanciales de la racionalidad científica" (Bartolucci, 2017, p. 13).

$\mathrm{Al}$ respecto, Ferreira (2008) destaca que la singularidad que se le atribuye al conocimiento científico es el resultado de la forma en que los cuerpos científicos ejecutan concretamente la ciencia; pero además, de cómo la aprenden y conciben para enseñarla a las demás personas.

Esto remite a los postulados de Popper (1977), quien aclara con respeto al propósito que se persigue que "la elección de tal finalidad tiene que ser, en última instancia, objeto de una decisión que vaya más allá de toda argumentación racional" (p. 37); esto alude a la individualidad del sujeto, asumida como la base sustantiva de los acuerdos y convenciones a los que da lugar la ciencia, alejados de la racionalidad que ella misma afirma contener. 
Como se aprecia, existe una clara definición de lo que significa la actividad científica en lo colectivo -social y antropológico-, no así en lo individual, que más allá de las modas y los rituales tribales que suelen acompañar el quehacer de quien investiga, se yergue como relevante al momento en que el sujeto científico enfrenta el reto cognitivo de explicar y comprender la realidad que dice conocer. En ello se aprecia una manifestación personal acerca de cómo se concibe la realidad, y de cómo se entiende para ser explicada. Por esto, a continuación se presenta un análisis de los principales sesgos psicológicos - cognitivos- y su importancia en la actividad científica investigativa, abordados ahora como sesgos conductuales.

\section{Basamento del ensayo}

Como su nombre lo indica, un sesgo cognitivo explica la tendencia a decantarse por una vía específica de pensamiento, condicionada por la intuición más que por el discernimiento. Se entiende como un mecanismo mediante el cual se llega rápidamente a una resolución, aunque ello implique cierto grado de superficialidad. En su mayoría se trata de heurísticos que comportan atajos cognitivos que predisponen a "adoptar actitudes ... epistémicamente fértiles" (Menna, 2014, p. 72). La idea es resolver rápido y bien. La mente del ser humano está gobernada principalmente por la acción de heurísticos irracionales (López-Astorga, 2011). Sobre esta base, los sesgos cognitivos nacen como una necesidad evolutiva del ser humano de producir juicios automáticos como mecanismo de respuesta inmediata del cerebro para asumir una posición rápida ante estímulos o situaciones particulares del entorno. Esto implica un filtraje subjetivo y selectivo de información, que conduce a decisiones y conductas erróneas en determinados contextos (Rodríguez, 2012).

Aunque puede parecer una actividad mental propia de las personas sin preparación, se muestran preferentemente en quienes, con un nivel de inteligencia mayor, como puede ser el caso de personal científico, quienes suelen asumir que cualquiera es más susceptible a cometer errores cognitivos que menos su grupo mismo (meta-sesgo). Esto conlleva a un grado de inconsciencia que no permite la introspección, el autoanálisis y la inteligencia, por lo que el error pasa inadvertido. 
Sobre esta base, se pasa por alto lo siguiente:

Sesgo es un error sistemático o una desviación de la verdad en los resultados. Esta desviación del valor verdad puede dar como resultado la sub/sobre-estimación de los efectos de una determinada intervención; sin embargo no es posible conocer hasta que [sic] grado han afectado los sesgos de los resultados en un estudio específico por ello es más apropiado considerar el 'Riesgo de Sesgo'. (Alarcón-Palacios et al., 2015, p. 305)

Situado en el campo de la investigación científica, a los sesgos cognitivos se les suele denominar también como ilusiones inferenciales, en virtud de que "el razonamiento es un proceso que da lugar a un producto inferencial" (González-Navarro, 2011, p. 167).

Basándose en ello, se afirma que "parte de las aportaciones científicas de los últimos decenios se han basado en la revisión de clasificaciones aceptadas durante largo tiempo, que ahora son concebidas como un subproducto de sesgos cognitivos" (Mundó, 2010, p. 317). Por ello, el objetivo es plantear una primera revisión conceptual del trabajo de la persona científica como individuo, en busca de fundamentar nuevos problemas de investigación en este campo.

\section{Enfoque de la disertación}

Se fundamenta en un abordaje cualitativo, entendido como:

[Una] metodología propia de las ciencias sociales, particularmente de la educación entendida como un hecho social, y como tal dinámico, influenciado por la interrelación de factores endógenos y exógenos, que se constituyen en una fuente importante de información, cuya comprensión e interpretación posibilite la transformación de la realidad educativa existente, proyectada a lograr la calidad del hecho social de la educación. (Portilla, Rojas y Hernández, 2014, p. 86)

Metodología cuya finalidad "es la construcción de conocimiento sobre la realidad social, desde la particularidad de la perspectiva de quienes la originan y la viven" (Portilla, Rojas y Hernández, 2014, 
p.86), punto de vista hermenéutico que aborda lo real entendido como proceso cultural, desde el cual se asume toda representación subjetiva inherente al ser humano, que actúa a la vez como marco interpretativo de todas sus acciones, y que permite llegar hasta su comprensión desde el marco de sus propias vivencias, experiencias y sensaciones, cuya finalidad es crear formas de ser y actuar en el mundo de la vida (González-Agudelo, 2013).

La unidad de análisis fue el comportamiento psicológico irracional del sujeto investigador científico, asociado a sus prácticas dominantes.

\section{Desarrollo de la pesquisa}

Sobre esta base, a continuación se desglosan los principales sesgos cognitivos asociados a la labor del personal científico investigador, bajo una interpretación analítica asociada a sus prácticas dominantes.

Sesgo de confirmación. Este pensamiento se sustenta en aceptar, sin dudar, las pruebas que apoyan las ideas propias; muestra, a la vez, escepticismo con aquellas que son contrarias a estas, y las asume como parciales o con intereses distintos. En términos de la ciencia y la investigación, es común observar cómo las personas alinean los resultados obtenidos a partir de sus propias certidumbres, haciéndolos coincidir con las premisas de su saber (lo que Estupinyà [2017] denomina como cherry picking). Esto se muestra, frecuentemente, cuando las hipótesis al final no coinciden con los resultados, circunstancia en la que muchas investigaciones suelen acomodarlas teórica y conceptualmente para que se ajusten a ellos o viceversa. En otro sentido, se encuentra el ajuste que se realiza con respecto a los resultados y su interpretación, o en relación con los resultados y el marco teórico-conceptual que se tiene. Lo transcendente estriba en que, al final, el conocimiento generado es una parcialidad cognitiva; una subjetividad disfrazada de objetividad por quien investiga.

Efecto halo. Tiene lugar cuando un rasgo positivo de una persona llama poderosamente la atención, al hacer pensar que dicho atributo define la totalidad del personaje -generalización-. Por ejemplo, se tiende a pensar que un investigador destacado científicamente posee la facultad de tener la razón siempre, al grado de no poder contradecírsele conceptual o teóricamente. El disenso con él no está permitido. En ocasiones se llega al extremo de pensar que su criterio es la unidad de 
medida perfecta para la cuantificación y cualificación de los fenómenos objeto de estudio. "Nadie se atreve a decirle al rey que viaja desnudo". En otro sentido, puede verse este efecto en la forma en que noveles de la ciencia suelen fundamentar sus publicaciones - papers- con base en las referencias bibliográficas de estos personajes, lo que por sí mismo les confiere valor a sus disertaciones. De aquí se desprende otro sesgo observable: el sesgo de autoridad.

Efecto de encuadre. La propensión a proponer diferentes conclusiones dependiendo de cómo se presente la evidencia obtenida. En principio, esto parecería lo más adecuado, el problema es cuando el investigador o investigadora tiene ya una conclusión en mente y busca enmarcarla con los resultados. El ejemplo más conocido es la anécdota sobre Alexander Fleming, quien en 1928 tomó unas vacaciones del trabajo dentro del laboratorio del Hospital St. Mary, a su regreso se percató de que en unas placas, donde había estado cultivando una bacteria, Staphylococcus aureus, había crecido también un hongo, observó que en el lugar donde creció el hongo la bacteria había sido inhibida (el hongo contaminaba el cultivo). A partir de este hallazgo concluyente, infirió que dicho hongo tenía propiedades antibacterianas (resultados). Algunas veces quien investiga encuentra lo que no buscaba (Serendipia, del inglés serendipity) y es entonces cuando debe deducir los resultados (la evidencia).

Anclaje. Es la tendencia a considerar en demasía una referencia o información anterior. Es notoria la influencia que ciertas ideas o conceptos ejercen. Durante muchos años los principios newtonianos fueron la guía para diseñar e interpretar toda teoría en la física de los grandes cuerpos en el universo. En general, las teorías tradicionales, como es el caso de las propuestas por Jean Piaget y Lev Semiónovich Vygotski dentro del campo educativo, tienden a condicionar el pensamiento dentro del ámbito de la didáctica y el currículo. Sus grandes aportaciones tienen un efecto dual: por una parte son clarificadoras de los fenómenos objeto de estudio y, por otra, condicionan la aparición de nuevas propuestas (paradigmas o modelos). De manera recurrente se busca coincidir con la matriz ideológica conceptual que más poder explicativo tiene, a fin de dotar de validez los nuevos saberes, ello anula la aparición de renovados códigos de entendimiento.

Ilusión de control. Este es el fenómeno cognitivo más clásico dentro del campo de la investigación experimental. La tendencia a creer 
que es posible, mediante el control y la manipulación, gobernar o al menos influir decididamente en los hechos sobre los que al final no es posible actuar completamente. Se trata del denominado "dogma de la santa percepción", que solo es posible asumiendo que se puede observar sin error, o al menos con el mínimo fallo. Esta ilusión funge como una verdadera arma conceptual que sostiene las disputas que desde hace más de un siglo mantienen las denominadas ciencias duras y las identificadas como sociales o de la conducta. Los grupos científicos dentro de las áreas físico-biológicas descalifican, desde estos baluartes ideológicos, las disciplinas que no emplean su método de control y manipulación de lo "real". El método científico decimonónico y sus técnicas empírico-analíticas suelen avasallar al pensamiento complejo y sistémico, propio de las ciencias sociales y humanas.

Sesgo de sujeto experimentador. Alineado con la ilusión de control, se encuentra el sesgo que los sujetos experimentadores científicos suelen asumir, quienes a menudo observan, seleccionan y publican los datos que están de acuerdo con las expectativas previas al experimento, expresadas en el método empleado. Se parte de la falsa premisa de que la validez de los resultados y sus conclusiones radica en las técnicas empleadas, la precisión de los equipos usados, el entrenamiento mecánico que poseen los sujetos experimentadores y el culto a los procesos estadísticos de la información. Se refiere concretamente a la búsqueda de la perfección a través del método. Para este tipo de sujetos científicos lo importante es cómo se procedió en la investigación y en su interpretación, no el porqué de ello.

Sesgo de observación selectiva. El adagio reza: cada quien ve como puede ver. En términos de la investigación científica equivale a elegir mientras se rechaza. El pensamiento suele posarse firmemente sobre "la cosa", sobre "el objeto", desestimándose cualquier otro fenómeno observable. A partir de ahí todo es posible. Las interpretaciones derivadas de este sesgo selectivo no permiten apreciar evidencias que podrían estar presentes frente a quien investiga, lo que deriva en la denominada "ceguera de taller". Los equipos investigadores no se abren a nuevos abordajes conceptuales y metodológicos acotándose a sí mismos. En términos coloquiales: "cuando eres un martillo, todo el mundo te parece un clavo".

La correlación ilusoria. Parecida a la apofenia (encontrar patrones y conexiones en sucesos aleatorios o en datos que no tienen 
sentido). Es la tendencia a suponer que existe relación entre dos variables, aunque no haya datos o evidencia que lo confirmen (correlaciones espurias). Por ejemplo, la falacia post hoc ergo propter hoc (después de esto, por lo tanto, por causa de esto) que se ejemplifica cuando se establece una relación aparentemente lógica, pero sin sentido, como es el caso de considerar la reprobación escolar como causa directa de la deserción o abandono, sin saber si esto es realmente cierto; o admitir que un sistema de gestión de la calidad mejora el desempeño de docentes y estudiantes, solo porque se refiere a la calidad. Algunas veces se expresa de manera ex post-facto, como cuando se identifica a estudiantes de alto desempeño y se asume que se debe a la calidad de la enseñanza que se impartió o a las competencias docentes de sus maestros o maestras. A decir de Torres-Salazar (2018, p. 7), se trata de un "patrón de asociación cognitiva irracional", o bien "de la tendencia a sobreestimar la existencia de covariación entre dos eventos" (Rodríguez-Ferreiro y Barberia, 2018, p.1).

Ilusión de serie o apofenia. Ya mencionado anteriormente, este sesgo se basa en la observación de patrones donde no los hay. Se trata de relacionar en conjunto hechos que no están conectados. Por ejemplo, una investigadora social especializada en fenómenos de género, puede ver problemas de discriminación donde no los hay, identificando variables y asociándolas para construir una realidad que solo está en su mente.

Finalmente, tal como afirma Peña (2012), "las tareas rutinarias y repetitivas con frecuencia hacen que nuestro pensamiento siga estilos ortodoxos, en vez de favorecer el pensamiento lateral. A esto se le denomina pensamiento en línea vertical" (p. 809).

Como es posible deducir, los sesgos cognitivos no vienen solos, generalmente se imbrican al momento de expresarse en la mente y la conducta del sujeto científico investigador. Su patrón conductual, sin importar el área de conocimiento que cultive, está supeditado a estas formas irracionales de pensar y actuar, que más allá de estar consensuadas por una comunidad que las valida y fomenta en sus ritos de iniciación y desarrollo, deben ser sometidas a escrutinio y vigilancia reflexiva, sobre todo por quienes se dedican a estas actividades. En conceptos de la psicóloga Eleanor Rosch (1978, citada por Mundó, 2010): "toda categorización está sujeta a sesgos que precisan de una permanente autoconciencia metodológica del quehacer científico" (p. 317). 


\section{Conclusiones}

Desde estos contextos de actuación, donde las falacias lógicas y los sesgos cognitivos son la constante, es necesario brindar mayor atención a las actitudes que las personas despliegan, sobre todo cuando el quehacer cotidiano se centra en generar conocimiento.

Un investigador o investigadora, por más apego a lo científico que muestre, debe prestar atención a todos estos fenómenos conductuales, que si bien son de índole psicológica, también lo son, en gran medida, biológica y social. La naturaleza humana tiene la propensión innata a congregarse en grupos, asociándose para fines específicos, tal es la condición de las comunidades; las científicas no son la excepción.

Querer pertenecer a una comunidad en especial se convierte en un propósito sublimado, desde donde se busca afanosamente la congruencia necesaria para pertenecer a dicho sistema. Identificarse con la tribu tiene un costo en términos de pensamiento y razón, de acción y condicionamiento. En aras de este objetivo, el cerebro humano gusta de aplicar soluciones conocidas (y avaladas por la comunidad), ya que probar cosas nuevas, mediante el ensayo y error, implica una nueva inversión en tiempo y esfuerzo: el cerebro, si ya conoce una solución, se vuelve "ciego" a otras posibles soluciones. Se trata de una ilusión creada por cánones dictados al razonamiento mediante caminos ya conocidos de reflexión y procesamiento de información (illusion of understanding); dicho en otros términos: "creer que algo de naturaleza compleja es simple solo porque se dice entenderlo, significa que no se entendió después de todo". Al final se trata de la mente y la biología conspirando contra el razonamiento.

Pero no todo está perdido. Lo primero que debe hacerse es tomar nota de estas condiciones, y desde ahí, aceptar que como persona científica e investigadora se puede estar equivocada. El sujeto científico investigador debe controlar su ego, ya que lo importante no es tener siempre la razón, sino acercarse lo más posible a la verdad. Estupinyà (2017) lo refiere de la siguiente manera:

El problema quizás viene de no asumir que la percepción nos engaña, que la memoria no es de fiar, que las emociones del momento dirigen nuestros pensamientos, que un montón de sesgos cognitivos condicionan nuestras decisiones, y que lo más 
inteligente sería no aferrarnos demasiado a esas conclusiones, sino tomarlas como hipótesis e ir revisándolas en función de si los nuevos datos que vayamos adquiriendo las confirman o no. Mente abierta es la que duda, no la que cree. (p. 146)

Aunado a ello, es necesario no convertirse en una "densa sombra que todo lo cubre y anula". Esto supone tener apertura al disenso y al debate que ello implica. Las ideas de otras personas, aunque contradictorias, pueden ser una rica veta para la nueva reflexión.

En este tenor, y tratándose de la investigación, es conveniente cambiar de preguntas, para así forzarse a cambiar de respuestas. Hay investigaciones que al abordar distintos fenómenos lo hacen de la misma manera. Dicho en lenguaje coloquial: "pretenden cruzar el desierto en bicicleta, solo porque son muy buenos andando en ella".

Finalmente, lo mejor es siempre la autocrítica: ¿Se habrá hecho lo mejor para abordar el problema? ¿El marco teórico-conceptual del que se partió es el adecuado? ¿Existe evidencia que no se está considerando? ¿Las conclusiones no están sesgadas hacia lo que de antemano se sabía? ¿No se están cometiendo errores? ¿Qué se hizo bien, qué se pudo hacer mejor? ¿Los grupos pares evaluadores habrán hecho un buen trabajo de revisión crítica a los proyectos? En el papel del maestro o maestra que enseña ciencia: ¿El personal docente investigador está tratando de "clonarse" a partir de sus aprendices o tesistas? ¿Se les está dando a estudiantes-aprendices la suficiente libertad conceptual e ideológica para pensar y actuar en sus proyectos? Por aquí se puede empezar a combatir los sesgos cognitivos en el trabajo científico.

\section{Referencias}

Alarcón-Palacios, M., Ojeda-Gómez, R. C. Ticse-Huaricancha, I. L. y Cajachagua-Hilario, Katherine. (2015). Análisis crítico de ensayos clínicos aleatorizados: Riesgo de sesgo. Rev Estomatol Herediana, 25(4), 304-308. Recuperado de http://www.scielo.org.pe/ $\mathrm{pdf} / \mathrm{reh} / \mathrm{v} 25 \mathrm{n} 4 / \mathrm{a} 08 \mathrm{v} 25 \mathrm{n} 4 . \mathrm{pdf}$

Bartolucci, J. (2017). La ciencia como problema sociológico. Sociológica, 32(92), 9-40. Recuperado de http://www.scielo.org.mx/pdf/ soc/v32n92/2007-8358-soc-32-92-00009.pdf 
Bourdieu, P. (2000). Los usos sociales de la ciencia. Buenos Aires: Ediciones Nueva Visión.

Estupinyà, P. (2017). La ciencia es coger cerezas con los ojos cerrados. MÈTODE, 92, 146-147. Recuperado de https://metode.cat/ wp-content/uploads/2017/02/92ES-estupinya-cherry-picking. pdf?.ga=2.171273191.63276025.1530942173-1214600858.1530942173

Ferreira, M. (2007a). Antropología de la ciencia. Una investigación autobservacional del proceso de formación de los científicos. Revista de Antropología Experimental, 7(4), 39-62. Recuperado de https://revistaselectronicas.ujaen.es/index.php/rae/article/ viewFile/2024/1772

Ferreira, M. A (2007b). La sociología del conocimiento científico: Una perspectiva crítica de futuro. Nómadas. Revista Crítica de Ciencias Sociales y Jurídicas, 16, 231-234. Recuperado de https:// webs.ucm.es/info/nomadas/16/mferreira.pdf

Ferreira, M. A. (2008). El conocimiento científico como actividad. Una aproximación sociológica a un sujeto singular. Nómadas. Revista Crítica de Ciencias Sociales y Jurídicas 19, 245-260. Recuperado de https://webs.ucm.es/info/nomadas/19/mferreira.pdf

González-Agudelo, E. M. (2013). Acerca del estado de la cuestión o sobre un pasado reciente en la investigación cualitativa con enfoque hermenéutico. Unipluriversidad, 13(1), 60-63. Recuperado de http://bibliotecadigital.udea.edu.co/bitstream/10495/3313/1/ GonzalesElvia_2013_estadocuestioninvestigacioncualitativa.pdf González-Navarro, M. (2011). Epistemología, razonamiento y cognición en el debate historiográfico constructivismo vs. reconstructivismo. Universitas Philosophica, 28(57), 163-187. Recuperado de http://www.scielo.org.co/pdf/unph/v28n57/v28n57a07.pdf

López-Astorga, M. (2011). Heurísticos y racionalidad: ¿La ignorancia beneficia a los estudiantes en algún sentido? Educar em Revista, Curitiba, Brasil, 41, 215-229. Recuperado de http://www.scielo. br/pdf/er/n41/14.pdf

Menna, S. H. (2014). Heurísticas y metodología de la ciencia. Mundo Siglo XXI, Revista del CIECAS-IPN, 9(32), 67-77. Recuperado de http://www.mundosigloxxi.ipn.mx/pdf/v09/32/06.pdf

Mundó, J. (2010). Categorías, sesgos y metaconcepciones en la metodología de la ciencia. Ludus Vitalis, 18(33), 317-320. Recuperado de http://diposit.ub.edu/dspace/bitstream/2445/69555/1/574519.pdf 
Peña. G. L. (2012). La naturaleza dual del proceso diagnóstico y su vulnerabilidad a los sesgos cognitivos. Rev Med Chile, 140, 806810. Recuperado de https://scielo.conicyt.cl/pdf/rmc/v140n6/ art17.pdf

Popper, K. (1977). La lógica de la investigación científica. Madrid: Tecnos.

Portilla, Ch. M. A., Rojas, Z. F. y Hernández, A. I. (2014). Investigación cualitativa: Una reflexión desde la educación como hecho social. Universitaria, 3(2), 86-100. Recuperado de http://revistas.udenar.edu.co/index.php/duniversitaria/article/view/2192/pdf_34

Rodríguez, Q. E. (2012). Toma de decisiones: La economía conductual. (Tesis de maestría). Universidad de Oviedo, España. Recuperado de http://digibuo.uniovi.es/dspace/bitstream/10651/13074/1/Trabajo\%20fin\%20de\%20m\%C3\%A1ster\%20Eduardo\%20Rodr\%C3\%ADguez\%20Quintana.pdf

Rodríguez-Ferreiro, J. y Barberia, I. (2018). Sesgos cognitivos y convicciones morales. Ciencia Cognitiva, 12(1), 8-10. Recuperado de http://medina-psicologia.ugr.es/cienciacognitiva/?p=1576

Rosch, E. (1978). Principles of categorization. In E. Rosch \& B. B. Lloyd (Eds.), Cognition and categorization. Hillsdale, NJ: Erlbaum. Reprinted in Margolis, E. and Laurence, S. (Eds.) (1999). Concepts: Core readings. Cambridge, MA: MIT Press.

Torres-Salazar, C. A. (2018). Sesgos cognitivos y su relación con el bienestar psicológico en estudiantes universitarios de la ciudad de Ambato (Tesis de licenciatura). Escuela de Psicología, Ambato, Ecuador. Recuperado de http://repositorio.pucesa.edu.ec/ bitstream/123456789/2172/1/76594.pdf

Villarruel-Fuentes, M. (2013). Ciencia globalizada y educación: Tendencias y marcos interpretativos para su desarrollo. Revista Iberoamericana de las Ciencias Sociales y Humanísticas, 2(4), 1-34. Recuperado de http://www.ricsh.org.mx/index.php/RICSH/ article/view/19/79 